\title{
Mass-dependence of self-diffusion coefficients in disparate-mass binary fluid mixtures*
}

\author{
I. Binas, I. Mryglod \\ Institute for Condensed Matter Physics of National Academy of Sciences of Ukraine, \\ 1 Svientsitskii Str., 79011 Lviv, Ukraine
}

Received June 24, 2009, in final form September 9, 2009

Self-diffusion coefficients of a binary fluid mixture with components differing only in their particle masses are studied, in particular the case when mass ratio $\mu$ of light and heavy particles tends to zero. These coefficients were calculated within the memory function formalism, using the systematic subsequence of approximations for the relaxation times of velocity autocorrelation function. We obtained a general relation for the self-diffusion coefficients which show polynomial dependence on the mass ratio $\mu$. The obtained expression has a correct Brownian limit. We developed the hierarchy of approximations for the self-diffusion coefficients that tends to an exact result from above and below when the order of approximations increases.

Key words: self-diffusion, mass asymmetry, binary fluid, Brownian limit

PACS: $66.10 .-x$

\section{Introduction}

Binary fluids with strong asymmetry in masses, sizes or charges of particles, forming a mixture present a good example of the system with complicated multiscale dynamics and have been the subject of numerous studies during the last years [1-14]. As a result of asymmetry, special features of such mixtures are revealed: fast sound, anomalous diffusion, dynamic arrest and cage effect, crossover to Brownian limit, etc. Some of these features can be already observed by the behaviour of self-diffusion. In particular, it was found [7] that

$$
\frac{D\left(m_{2}\right)}{D\left(m_{1}\right)} \sim\left(\frac{m_{1}}{m_{2}}\right)^{\kappa},
$$

where $D\left(m_{2}\right)$ and $D\left(m_{1}\right)$ are the self-diffusion coefficients of heavy and light particles respectively, $m_{2}$ and $m_{1}$ are masses. The coefficient $\kappa$ was evaluated in various approaches which provide substantially different results.

For example, in the kinetic theory of gasses this coefficient is equal to $1 / 2$. However, molecular dynamics (MD) simulations have shown that in the condensed state the ratio of self-diffusion coefficients varies more weakly with mass ratio. For equimolar systems, the change of the mass of one species has nearly the same effect on the self-diffusion coefficient of both species [1]. Of course, there is some difference in mass-dependence in favor of the species whose mass is changing.

Extensive MD simulations for equimolar mixtures of Lennard-Jones (LJ) isotopes [7] showed that $\kappa$ varies from 0.06 to 0.1 . However, computations for much wider ranges of mass ratio $\mu=$ $m_{1} / m_{2}$ and ratio of light $\left(N_{1}\right)$ and heavy $\left(N_{2}\right)$ particle numbers showed that relation $(1)$ is too raw. In particular, the results for mass ratios changing from 1 to 0.02 for LJ and Weeks-ChandlerAnderson (WCA) fluids [1], state that the self-diffusion coefficient of the Brownian particle with mass ratio less than 0.04 reaches a thermodynamic limit depending neither on its mass nor on the number of light particles. The same qualitative behaviour was obtained in a range of other works (see for example $[2,3,5]$ and references therein).

*Dedicated to the 100th birthday of Prof. M.M. Bogolyubov. 
A number of researches provided by Tankeshwar et al. [8-10] showed that in case of a tracer diffusion $\left(N_{2}=1\right)$ a relation takes place

$$
\frac{D\left(m_{2}\right)}{D\left(m_{1}\right)} \simeq \sqrt{\frac{1+m_{1} / m_{2}}{2}} .
$$

Still, this result is not precise enough because of a range of approximations. The most crude of them are: (i) the Kirkwood superposition approximation used in describing the three-body contribution to the fourth-order sum rules and (ii) unreasonable neglecting of some terms in the expression for the self-diffusion coefficient. These lead to a simple relation (2), which is the same for Mori approximations of the second and the third order (see definition in the next section). This means that the result cannot be improved by using higher-order approximations and hence it remains not close enough to the exact solution.

The goal of our work is to study analytically the mass dependence of the self-diffusion coefficient. We avoid using any approximations or perform any MD calculations until this is necessary. The case of Brownian regime and mechanism of changing $\kappa$ from 0.5 to 0 in equation (1) is of particular interest to us.

The paper is organized as follows. In the next section we provide theoretical background for the approximation scheme we use. Then, we give the main points in derivation of mass dependence of the self-diffusion coefficients, and subsequently we present an analysis of the obtained results. Finally, we end with a conclusion and a short outlook.

\section{Theoretical background}

\subsection{Mori memory function formalism}

The general Green-Kubo relation [16] for the self-diffusion coefficient $D_{i}$ is given as

$$
D=\frac{k_{\mathrm{B}} T}{m} \int_{0}^{\infty} \psi(t) \mathrm{d} t,
$$

where $k_{\mathrm{B}}$ and $T$ are the Boltzmann constant and the temperature of the system, respectively; $\psi(t)$ are the normalized velocity autocorrelation functions (VACFs) defined as

$$
\psi(t)=\frac{1}{3 N} \sum_{j=1}^{N}\left\langle\mathbf{v}_{j}(t) \mathbf{v}_{j}(0)\right\rangle .
$$

Since the exact evaluation of VACFs is not yet available, they can be calculated directly in molecular dynamics simulations. This way presents a popular and widely available approach to getting desirable quantities for a wide range of input data. But still MD results obtained in that way do not provide us with direct mass-dependence of self-diffusion coefficients. Subsequent approximation models need to be used to describe the behaviour of self-diffusion. This causes additional distortion of the real form of the dependence between the observed quantities. Also, MD results fall under the finite-size effect $[5,12,17]$, when in the limit of big mass of heavy particles finite-size correction should be applied.

Another way of approximating the VACFs is to use the memory function formalism. Mori showed [18] at microscopic level that $\psi(t)$ satisfies the equation

$$
\frac{\mathrm{d} \psi(t)}{\mathrm{d} t}+\int_{0}^{t} \varphi_{1}(t-\tau) \psi(\tau) \mathrm{d} \tau=0
$$

where $\varphi_{1}(t)$ is the first-order memory function, which in its turn satisfies the same equation with $\varphi_{2}(t)$ being its memory function and so on. 
An important property of the memory function equation is the continued fraction representation for the Laplace transform of the VAC function:

$$
\widetilde{\psi}(z)=\frac{1}{z+\widetilde{\varphi}_{1}(z)}=\frac{1}{z+\frac{\Gamma_{1}}{z+\widetilde{\varphi}_{2}(z)}}=\cdots=\frac{1}{z+\frac{\Gamma_{1}}{z+\cdots \frac{\Gamma_{n}}{z+\widetilde{\varphi}_{n+1}(z)}}}
$$

and

$$
\widetilde{\varphi}_{n}(z)=\frac{\Gamma_{n}}{z+\widetilde{\varphi}_{n+1}(z)}, \quad n=1,2, \ldots,
$$

where $\Gamma_{n}=\varphi_{n}(0)$ are initial values of the $n$th stage of the memory function $\varphi_{n}(t)$ and are called Mori coefficients. Such representation provides a guide for a systematic development of model computations of the VAC functions.

With the help of the first-order memory function, the self-diffusion coefficient can now be presented as follows

$$
D=\frac{k_{\mathrm{B}} T}{m} \frac{1}{\widetilde{\varphi}_{1}(0)}
$$

\subsection{Mori coefficients and frequency sum rules}

The first three Mori coefficients $\Gamma_{1}-\Gamma_{3}$ are [19]

$$
\begin{gathered}
\Gamma_{1}(0)=U_{1}, \\
\Gamma_{2}(0)=\frac{U_{2}}{\Gamma_{1}}-\Gamma_{1}, \\
\Gamma_{3}(0)=\frac{U_{3}}{\Gamma_{1} \Gamma_{2}}-\frac{U_{2}}{\Gamma_{1} \Gamma_{2}}\left(\Gamma_{1}+\Gamma_{2}\right) .
\end{gathered}
$$

Here

$$
U_{n}=\frac{1}{N}\left\langle\sum_{i=1}^{N} \mathbf{v}_{i}^{(n)} \mathbf{v}_{i}^{(n)}\right\rangle
$$

is the ensemble average of the $N$ particle contribution; $\mathbf{v}_{i}^{(n)}$ denotes the $n$th time derivative of the normalized velocities. Note that the coefficients $U_{1}(m), U_{2}(m)$ and $U_{3}(m)$ are the second-, fourth-, and sixth-frequency sum rules in the short time expansion of the VAC function

$$
\psi(t)=1-U_{1} \frac{t^{2}}{2 !}+U_{2} \frac{t^{4}}{4 !}-U_{3} \frac{t^{6}}{6 !}+\cdots
$$

\subsection{Phenomenological forms of the memory function}

A number of phenomenological forms of the memory function have been proposed [16]. The most common of them is the Gaussian model which in general can be written as

$$
\varphi_{n}(t)=a \exp \left[-b^{2} t^{2} / 2\right]
$$

The parameters $a$ and $b$ can be determined by requiring that the first two coefficients in the expansion of the above equation and that of the exact memory function (8) are the same. In particular, in studies $[1,9,13] n=2, a=\Gamma_{1}$ and $b=\sqrt{\Gamma_{2}}$; and in the study [10] $n=3, a=\Gamma_{2}$ and $b=\sqrt{\Gamma_{3}}$.

Since according to the Bogolyubov's hypothesis of relaxation time hierarchy [15] the higher order memory functions $\varphi_{n}$ in continued fraction representation (3) are expected to converge, we assume that for some $s$, there takes place the equality

$$
\widetilde{\varphi}_{s+1}(0)=\lambda \widetilde{\varphi}_{s}(0)
$$


where $\lambda$ is a fitting parameter. Substituting equation (9) into a continued fraction representation (3) we obtain a range of approximations for $\widetilde{\varphi}_{1}(0)$ which in general can be written as follows

$$
\widetilde{\varphi}_{1}(0)=\left(\prod_{l=1}^{\left[\frac{n}{2}\right]} \frac{\Gamma_{2 l-1}}{\Gamma_{2 l}}\right) \cdot \begin{cases}\sqrt{\frac{\Gamma_{n+1}}{\lambda}} & \text { if } n \text { is even } \\ \frac{\sqrt{\lambda} \Gamma_{n}}{\sqrt{\Gamma_{n+1}}} & \text { if } n \text { is odd }\end{cases}
$$

As can be seen, representation (10) coincides with the Gaussian form for $n=2$ and $n=3$ when $\lambda=\pi / 2$.

\section{Expressions for the self-diffusion coefficient}

\subsection{Frequency sum rules}

We consider a two-component mixture consisting of $N=N_{1}+N_{2}$ particles, where $N_{1}$ is a number of light particles of mass $m_{1}$, and $N_{2}$ is a number of heavy particles of mass $m_{2}$. Coefficient $\mu=\left(m_{1} / m_{2}\right)<1$ denotes the mass ratio of light and heavy particles. We assume that the interaction potential depends only on the distance between particles and does not depend on the sort of a particle. Hence, $U_{n}\left(m_{1}\right)$ can be found as

$$
U_{n}\left(m_{1}\right)=\frac{1}{N_{1}}\left\langle\sum_{i=1}^{N_{1}} \mathbf{v}_{i}^{(n)} \mathbf{v}_{i}^{(n)}\right\rangle=\left\langle\left[\mathbf{v}_{1}^{(n)}\right]^{2}\right\rangle,
$$

where the 1st particle is assumed to be of mass $m_{1}$. Also we consider the case of homogeneous and isotropic fluids so that all the directions (x, y, z) are equivalent. Hence, instead of calculating $\left\langle\left[v_{1}^{(n)}\right]^{2}\right\rangle$, we calculate $\left\langle\left[v_{1 x}^{(n)}\right]^{2}\right\rangle$.

In the present work we derive expressions for $U_{1}, U_{2}$ and $U_{3}$ in which explicit mass-dependence is drawn out. For this purpose, we present time derivatives of the normalized velocities via potential derivatives.

Assume that the particle with index 1 is a light particle. Then its velocity derivatives can be found as

$$
\begin{aligned}
\dot{v}_{1 x}= & -\frac{1}{m_{1}} \sum_{j=1}^{N} \frac{\partial U_{1 j}}{\partial r_{1 x}} \\
\ddot{v}_{1 x}= & -\frac{1}{m_{1}} \sum_{j=1}^{N} \frac{\partial^{2} U_{1 j}}{\partial r_{1 x} \partial r_{1 \alpha}}\left(\dot{r}_{1 \alpha}-\dot{r}_{j \alpha}\right)=-\frac{1}{m_{1}} \sum_{j=1}^{N} \frac{\partial^{2} U_{1 j}}{\partial r_{1 x} \partial r_{1 \alpha}}\left(v_{1 \alpha}-v_{j \alpha}\right) \\
= & -\frac{1}{m_{1}} \sum_{j=1}^{N_{1}} \frac{\partial^{2} U_{1 j}}{\partial r_{1 x} \partial r_{1 \alpha}} v_{1 \alpha}+\frac{1}{m_{1}} \sum_{j=1}^{N_{1}} \frac{\partial^{2} U_{1 j}}{\partial r_{1 x} \partial r_{1 \alpha}} v_{j \alpha}-\frac{1}{m_{1}} \sum_{k=1}^{N_{2}} \frac{\partial^{2} U_{1 k}}{\partial r_{1 x} \partial r_{1 \alpha}} v_{1 \alpha} \\
& +\frac{1}{m_{1}} \sum_{k=1}^{N_{2}} \frac{\partial^{2} U_{1 k}}{\partial r_{1 x} \partial r_{1 \alpha}} v_{k \alpha}, \\
v_{1 x}^{(3)}= & -\frac{1}{m_{1}} \sum_{j=1}^{N} \frac{\partial^{3} U_{1 j}}{\partial r_{1 x} \partial r_{1 \alpha} \partial r_{1 \beta}}\left(v_{1 \alpha}-v_{j \alpha}\right)\left(v_{1 \beta}-v_{j \beta}\right)-\frac{1}{m_{1}} \sum_{j=1}^{N} \frac{\partial^{2} U_{1 j}}{\partial r_{1 x} \partial r_{1 \alpha}}\left(\dot{v}_{1 \alpha}-\dot{v}_{j \alpha}\right) \\
= & -\frac{1}{m_{1}} \sum_{j=1}^{N} \frac{\partial^{3} U_{1 j}}{\partial r_{1 x} \partial r_{1 \alpha} \partial r_{1 \beta}}\left(v_{1 \alpha}-v_{j \alpha}\right)\left(v_{1 \beta}-v_{j \beta}\right) \\
& -\frac{1}{m_{1}} \sum_{j=1}^{N} \frac{\partial^{2} U_{1 j}}{\partial r_{1 x} \partial r_{1 \alpha}}\left(-\frac{1}{m_{1}} \sum_{l=1}^{N} \frac{\partial U_{1 l}}{\partial r_{1 \alpha}}+\frac{1}{m_{j}} \sum_{l=1}^{N} \frac{\partial U_{j l}}{\partial r_{j \alpha}}\right)
\end{aligned}
$$


After substituting (12) in the expression (11) we obtain

$$
U_{1}\left(m_{1}\right)=\frac{\left\langle\dot{v}_{1 x}^{2}\right\rangle}{\left\langle v_{1 x}^{2}\right\rangle}=\frac{1}{k_{\mathrm{B}} T m_{1}} \sum_{j, l=1}^{N}\left\langle\frac{\partial U_{1 j}}{\partial r_{1 x}} \frac{\partial U_{1 l}}{\partial r_{1 x}}\right\rangle .
$$

Another way of deriving the expression for $U_{1}\left(m_{1}\right)$ is to shift the time derivative in $\left\langle\dot{v}_{1 x}^{2}\right\rangle$ from the first to the second multiplier and to cancel all the terms which give zero on average, i. e. the terms like $\left\langle A(\mathbf{r}) v_{i \alpha} v_{j \beta}\right\rangle$ for $i \alpha \neq j \beta$ :

$$
\begin{aligned}
U_{1}\left(m_{1}\right) & =\frac{\left\langle\dot{v}_{1 x}^{2}\right\rangle}{\left\langle v_{1 x}^{2}\right\rangle}=-\frac{\left\langle v_{1 x} \ddot{v}_{1 x}\right\rangle}{\left\langle v_{1 x}^{2}\right\rangle}=\frac{1}{k_{\mathrm{B}} T} \sum_{j=1}^{N}\left\langle\frac{\partial^{2} U_{1 j}}{\partial r_{1 x} \partial r_{1 \alpha}}\left(v_{1 \alpha}-v_{j \alpha}\right) v_{1 x}\right\rangle \\
& =\frac{1}{k_{\mathrm{B}} T} \sum_{j=1}^{N}\left\langle\frac{\partial^{2} U_{1 j}}{\partial r_{1 x}^{2}} v_{1 x}^{2}\right\rangle=\frac{1}{m_{1}} \sum_{j=1}^{N}\left\langle\frac{\partial^{2} U_{1 j}}{\partial r_{1 x}^{2}}\right\rangle .
\end{aligned}
$$

Expressions for $U_{2}\left(m_{1}\right)$ and $U_{3}\left(m_{1}\right)$ are obtained in the same way and they read

$$
\begin{aligned}
& U_{2}\left(m_{1}\right)=\frac{1}{m_{1}^{2}}\left[\sum_{j, l=1}^{N_{1}}\left\langle\frac{\partial^{2} U_{1 j}}{\partial r_{1 x} \partial r_{1 \alpha}} \frac{\partial^{2} U_{1 l}}{\partial r_{1 x} \partial r_{1 \alpha}}\right\rangle+\sum_{j=1}^{N_{1}}\left\langle\left(\frac{\partial^{2} U_{1 j}}{\partial r_{1 x} \partial r_{1 \alpha}}\right)^{2}\right\rangle\right. \\
& +\sum_{k, m=1}^{N_{2}}\left\langle\frac{\partial^{2} U_{1 k}}{\partial r_{1 x} \partial r_{1 \alpha}} \frac{\partial^{2} U_{1 m}}{\partial r_{1 x} \partial r_{1 \alpha}}\right\rangle+ \\
& \left.+2 \sum_{j=1}^{N_{1}} \sum_{k=1}^{N_{2}}\left\langle\frac{\partial^{2} U_{1 j}}{\partial r_{1 x} \partial r_{1 \alpha}} \frac{\partial^{2} U_{1 k}}{\partial r_{1 x} \partial r_{1 \alpha}}\right\rangle\right]+\frac{1}{m_{1} m_{2}} \sum_{k=1}^{N_{2}}\left\langle\left(\frac{\partial^{2} U_{1 k}}{\partial r_{1 x} \partial r_{1 \alpha}}\right)^{2}\right\rangle, \\
& U_{3}\left(m_{1}\right)=\frac{1}{m_{1}^{3}}\left[6 k_{\mathrm{B}} T \sum_{j, l=1}^{N_{1}}\left\langle\frac{\partial^{3} U_{1 j}}{\partial r_{1 x} \partial r_{1 \alpha} \partial r_{1 \beta}} \frac{\partial^{3} U_{1 l}}{\partial r_{1 x} \partial r_{1 \alpha} \partial r_{1 \beta}}\right\rangle+6 k_{\mathrm{B}} T \sum_{j=1}^{N_{1}}\left\langle\left(\frac{\partial^{3} U_{1 j}}{\partial r_{1 x} \partial r_{1 \alpha} \partial r_{1 \beta}}\right)^{2}\right\rangle\right. \\
& +8 k_{\mathrm{B}} T \sum_{j=1}^{N_{1}} \sum_{k=1}^{N_{2}}\left\langle\frac{\partial^{3} U_{1 j}}{\partial r_{1 x} \partial r_{1 \alpha} \partial r_{1 \beta}} \frac{\partial^{3} U_{1 k}}{\partial r_{1 x} \partial r_{1 \alpha} \partial r_{1 \beta}}\right\rangle \\
& +3 k_{\mathrm{B}} T \sum_{k, m=1}^{N_{2}}\left\langle\frac{\partial^{3} U_{1 k}}{\partial r_{1 x} \partial r_{1 \alpha} \partial r_{1 \beta}} \frac{\partial^{3} U_{1 m}}{\partial r_{1 x} \partial r_{1 \alpha} \partial r_{1 \beta}}\right\rangle-4 \sum_{j=1}^{N_{1}}\left\langle\frac{\partial^{3} U_{1 j}}{\partial r_{1 x} \partial r_{1 \alpha}^{2}} s_{1}\left(m_{1}\right)\right\rangle \\
& \left.-2 \sum_{k=1}^{N_{2}}\left\langle\frac{\partial^{3} U_{1 k}}{\partial r_{1 x} \partial r_{1 \alpha}^{2}} s_{1}\left(m_{1}\right)\right\rangle+\frac{1}{k_{\mathrm{B}} T}\left\langle s_{1}^{2}\left(m_{1}\right)\right\rangle\right] \\
& +\frac{1}{m_{1}^{2} m_{2}}\left[4 k_{\mathrm{B}} T \sum_{j=1}^{N_{1}} \sum_{k=1}^{N_{2}}\left\langle\frac{\partial^{3} U_{1 j}}{\partial r_{1 x} \partial r_{1 \alpha}^{2}} \frac{\partial^{3} U_{1 k}}{\partial r_{1 x} \partial r_{1 \beta}^{2}}\right\rangle+2 k_{\mathrm{B}} T \sum_{k, m=1}^{N_{2}}\left\langle\frac{\partial^{3} U_{1 k}}{\partial r_{1 x} \partial r_{1 \alpha}^{2}} \frac{\partial^{3} U_{1 m}}{\partial r_{1 x} \partial r_{1 \beta}^{2}}\right\rangle\right. \\
& +4 k_{\mathrm{B}} T \sum_{k=1}^{N_{2}}\left\langle\left(\frac{\partial^{3} U_{1 k}}{\partial r_{1 x} \partial r_{1 \alpha} \partial r_{1 \beta}}\right)^{2}\right\rangle+4 \sum_{j=1}^{N_{1}}\left\langle\frac{\partial^{3} U_{1 j}}{\partial r_{1 x} \partial r_{1 \alpha}^{2}} s_{2}\left(m_{1}\right)\right\rangle \\
& \left.-2 \sum_{k=1}^{N_{2}}\left\langle\frac{\partial^{3} U_{1 k}}{\partial r_{1 x} \partial r_{1 \alpha}^{2}}\left(s_{1}\left(m_{1}\right)-s_{2}\left(m_{1}\right)\right)\right\rangle-\frac{2}{k_{\mathrm{B}} T}\left\langle s_{1}\left(m_{1}\right) s_{2}\left(m_{1}\right)\right\rangle\right] \\
& +\frac{1}{m_{1} m_{2}^{2}}\left[k_{\mathrm{B}} T \sum_{k, m=1}^{N_{2}}\left\langle\frac{\partial^{3} U_{1 k}}{\partial r_{1 x} \partial r_{1 \alpha}^{2}} \frac{\partial^{3} U_{1 m}}{\partial r_{1 x} \partial r_{1 \beta}^{2}}\right\rangle+2 k_{\mathrm{B}} T \sum_{k=1}^{N_{2}}\left\langle\left(\frac{\partial^{3} U_{1 k}}{\partial r_{1 x} \partial r_{1 \alpha} \partial r_{1 \beta}}\right)^{2}\right\rangle\right. \\
& \left.+2 \sum_{k=1}^{N_{2}}\left\langle\frac{\partial^{3} U_{1 k}}{\partial r_{1 x} \partial r_{1 \alpha}^{2}} s_{2}\left(m_{1}\right)\right\rangle+\frac{1}{k_{\mathrm{B}} T}\left\langle s_{2}^{2}\left(m_{1}\right)\right\rangle\right] \text {, }
\end{aligned}
$$


where

$$
\begin{aligned}
s_{1}\left(m_{1}\right)= & \sum_{j, l=1}^{N_{1}} \frac{\partial^{2} U_{1 j}}{\partial r_{1 x} \partial r_{1 \alpha}}\left(\frac{\partial U_{1 l}}{\partial r_{1 \alpha}}-\frac{\partial U_{j l}}{\partial r_{j \alpha}}\right)+\sum_{j=1}^{N_{1}} \sum_{k=1}^{N_{2}} \frac{\partial^{2} U_{1 j}}{\partial r_{1 x} \partial r_{1 \alpha}}\left(\frac{\partial U_{1 k}}{\partial r_{1 \alpha}}-\frac{\partial U_{j k}}{\partial r_{j \alpha}}\right) \\
& +\sum_{j=1}^{N_{1}} \sum_{k=1}^{N_{2}} \frac{\partial^{2} U_{1 k}}{\partial r_{1 x} \partial r_{1 \alpha}} \frac{\partial U_{1 j}}{\partial r_{1 \alpha}}+\sum_{k, m=1}^{N_{2}} \frac{\partial^{2} U_{1 k}}{\partial r_{1 x} \partial r_{1 \alpha}} \frac{\partial U_{1 m}}{\partial r_{1 \alpha}} \\
s_{2}\left(m_{1}\right)= & \sum_{j=1}^{N_{1}} \sum_{k=1}^{N_{2}} \frac{\partial^{2} U_{1 k}}{\partial r_{1 x} \partial r_{1 \alpha}} \frac{\partial U_{k j}}{\partial r_{k \alpha}}+\sum_{k, m=1}^{N_{2}} \frac{\partial^{2} U_{1 k}}{\partial r_{1 x} \partial r_{1 \alpha}} \frac{\partial U_{k m}}{\partial r_{k \alpha}}
\end{aligned}
$$

Expressions for $U_{1}\left(m_{2}\right), U_{2}\left(m_{2}\right)$ and $U_{3}\left(m_{2}\right)$ can be obtained from expressions (13)-(15) by interchanging $m_{1}$ by $m_{2}$ and sums over $N_{1}$ by sums over $N_{2}$.

In general, frequency sum rules can be presented as

$$
\begin{gathered}
U_{1}\left(m_{1}\right)=\frac{1}{m_{1}} A_{1}, \\
U_{1}\left(m_{2}\right)=\frac{1}{m_{2}} A_{1}, \\
U_{2}\left(m_{1}\right)=\frac{1}{m_{1}^{2}} B_{1}+\frac{1}{m_{1} m_{2}} B_{2}, \\
U_{2}\left(m_{2}\right)=\frac{1}{m_{2}^{2}} \widetilde{B}_{1}+\frac{1}{m_{1} m_{2}} \widetilde{B}_{2}, \\
U_{3}\left(m_{1}\right)=\frac{1}{m_{1}^{3}} C_{1}+\frac{1}{m_{1}^{2} m_{2}} C_{2}+\frac{1}{m_{1} m_{2}^{2}} C_{3}, \\
U_{3}\left(m_{2}\right)=\frac{1}{m_{2}^{3}} \widetilde{C}_{1}+\frac{1}{m_{1} m_{2}^{2}} \widetilde{C}_{2}+\frac{1}{m_{1}^{2} m_{2}} \widetilde{C}_{3},
\end{gathered}
$$

where coefficients $A_{1}, \ldots, \widetilde{C}_{3}$ depend on the ensemble average of the partial derivatives of the interaction potential and do not depend on the masses of particles.

\subsection{Mori coefficients}

Now the Mori coefficients can be found according to relations (5)-(7):

$$
\begin{gathered}
\Gamma_{1}\left(m_{1}\right)=U_{1}\left(m_{1}\right)=\frac{1}{m_{1}} A_{1}, \\
\Gamma_{1}\left(m_{2}\right)=\frac{1}{m_{2}} A_{1}=\frac{1}{m_{1}} \mu A_{1}=\mu \Gamma_{1}\left(m_{1}\right), \\
\Gamma_{2}\left(m_{1}\right)=\frac{1}{m_{1}} \frac{B_{1}-A_{1}^{2}}{A_{1}}\left[1+\mu \frac{B_{2}}{B_{1}-A_{1}^{2}}\right]=\frac{1}{m_{1}} K_{0}\left[1+\mu K_{1}\right], \\
\Gamma_{2}\left(m_{2}\right)=\frac{1}{m_{1}} \frac{\widetilde{B}_{2}}{A_{1}}\left[1+\mu\left(\frac{\widetilde{B}_{1}-A_{1}^{2}}{\widetilde{B}_{2}}\right)\right]=\frac{1}{m_{1}} \widetilde{K}_{0}\left[1+\mu \widetilde{K}_{1}\right] .
\end{gathered}
$$


For the next coefficients $\Gamma_{3}\left(m_{1}\right)$ and $\Gamma_{3}\left(m_{2}\right)$ we assume that $\mu$ is small enough so we can use Taylor expansion to simplify the coefficients and to present them in the form of polynomial:

$$
\begin{aligned}
\Gamma_{3}\left(m_{1}\right)= & \frac{1}{m_{1}} \frac{A_{1} C_{1}-B_{1}^{2}}{A_{1}\left(B_{1}-A_{1}^{2}\right)} \frac{1+\mu \frac{A_{1} C_{2}-2 B_{1} B_{2}}{A_{1} C_{1}-B_{1}^{2}}+\mu^{2} \frac{A_{1} C_{3}-B_{2}^{2}}{A_{1} C_{1}-B_{1}^{2}}}{1+\mu \frac{B_{2}}{B_{1}-A_{1}^{2}}} \simeq \frac{1}{m_{1}} \frac{A_{1} C_{1}-B_{1}^{2}}{A_{1}\left(B_{1}-A_{1}^{2}\right)} \\
& \times\left[1+\mu\left(\frac{A_{1} C_{2}-2 B_{1} B_{2}}{A_{1} C_{1}-B_{1}^{2}}-\frac{B_{2}}{B_{1}-A_{1}^{2}}\right)\right. \\
& \left.+\mu^{2}\left(\frac{A_{1} C_{3}-B_{2}^{2}}{A_{1} C_{1}-B_{1}^{2}}-\frac{B_{2}}{B_{1}-A_{1}^{2}}\left(\frac{A_{1} C_{2}-2 B_{1} B_{2}}{A_{1} C_{1}-B_{1}^{2}}-\frac{B_{2}}{B_{1}-A_{1}^{2}}\right)\right)\right] \\
\Gamma_{3}\left(m_{2}\right) \simeq & \frac{1}{m_{1}} L_{0}\left[1+\mu L_{1}+\mu^{2} L_{2}\right], \\
& \frac{1}{m_{1}} \frac{A_{1} \widetilde{C}_{3}-\widetilde{B}_{2}^{2}}{A_{1} \widetilde{B}_{2}}\left[1+\mu\left(\frac{A_{1} \widetilde{C}_{2}-2 \widetilde{B}_{1} \widetilde{B}_{2}}{A_{1} \widetilde{C}_{3}-\widetilde{B}_{2}^{2}}-\frac{\widetilde{B}_{1}-A_{1}^{2}}{\widetilde{B}_{2}}\right)+\right. \\
& \left.+\mu^{2}\left(\frac{A_{1} \widetilde{C}_{1}-\widetilde{B}_{1}^{2}}{A_{1} \widetilde{C}_{3}-\widetilde{B}_{2}^{2}}-\frac{\widetilde{B}_{1}-A_{1}^{2}}{\widetilde{B}_{2}}\left(\frac{A_{1} \widetilde{C}_{2}-2 \widetilde{B}_{1} \widetilde{B}_{2}}{A_{1} \widetilde{C}_{3}-\widetilde{B}_{2}^{2}}-\frac{\widetilde{B}_{1}-A_{1}^{2}}{\widetilde{B}_{2}}\right)\right)\right] \\
= & \frac{1}{m_{1}} \widetilde{L}_{0}\left[1+\mu \widetilde{L}_{1}+\mu^{2} \widetilde{L}_{2}\right] \cdot
\end{aligned}
$$

\subsection{Self-diffusion coefficients}

The first three approximations for the self-diffusion coefficients follow from equations (4) and (10) using expressions for $\Gamma_{n}$ obtained in the previous subsection. Expressions for the first approximation are

$$
\begin{aligned}
& D\left(m_{1}\right)=\frac{k_{\mathrm{B}} T}{m_{1}} \sqrt{\lambda} \frac{1}{\sqrt{A_{1} / m_{1}}}=\frac{1}{\sqrt{m_{1}}} \frac{k_{\mathrm{B}} T \sqrt{\lambda}}{\sqrt{A_{1}}} \\
& D\left(m_{2}\right)=\frac{1}{\sqrt{m_{2}}} \frac{k_{\mathrm{B}} T \sqrt{\lambda}}{\sqrt{A_{1}}} .
\end{aligned}
$$

Now the ratio of these coefficients is equal to

$$
\frac{D\left(m_{2}\right)}{D\left(m_{1}\right)}=\sqrt{\frac{m_{1}}{m_{2}}} .
$$

As can be seen, it coincides with the kinetic theory results, but does not give good mass-dependence in the Brownian limit. Correct qualitative behaviour is obtained already in the second approximation:

$$
\begin{aligned}
D\left(m_{1}\right) & =\frac{k_{\mathrm{B}} T}{m_{1}} \frac{\sqrt{K_{0} / m_{1}\left[1+\mu K_{1}\right]}}{\sqrt{\lambda} A_{1} / m_{1}} \simeq \frac{1}{\sqrt{m_{1}}} \frac{k_{\mathrm{B}} T \sqrt{K_{0}}}{\sqrt{\lambda} A_{1}}\left[1+\frac{1}{2} \mu K_{1}-\frac{1}{8} \mu^{2} K_{1}^{2}\right]+O\left(\mu^{3}\right) \\
D\left(m_{2}\right) & \simeq \frac{1}{\sqrt{m_{1}}} \frac{k_{\mathrm{B}} T \sqrt{\widetilde{K}_{0}}}{\sqrt{\lambda} A_{1}}\left[1+\frac{1}{2} \mu \widetilde{K}_{1}-\frac{1}{8} \mu^{2} \widetilde{K}_{1}^{2}\right]+O\left(\mu^{3}\right) \\
\frac{D\left(m_{2}\right)}{D\left(m_{1}\right)} & =\sqrt{\frac{\widetilde{K}_{0}}{K_{0}}}\left[1+\frac{1}{2} \mu\left(\widetilde{K}_{1}-K_{1}\right)-\frac{1}{8} \mu^{2}\left(\widetilde{K}_{1}-K_{1}\right)\left(\widetilde{K}_{1}+3 K_{1}\right)\right]+O\left(\mu^{3}\right)
\end{aligned}
$$

Here we obtain polynomial mass-dependence for $\mu<1$ which vanishes in the limit $\mu \rightarrow 0$ :

$$
\lim _{\mu \rightarrow 0} \frac{D\left(m_{2}\right)}{D\left(m_{1}\right)}=\sqrt{\frac{\widetilde{K}_{0}}{K_{0}}}=\sqrt{\frac{\widetilde{B}_{2}}{B_{1}-A_{1}^{2}}} .
$$


The third approximation does not change the qualitative behaviour of the expressions whereas it refines the coefficients next to $\mu^{n}$ :

$$
\begin{aligned}
D\left(m_{1}\right) & \simeq \frac{1}{m_{1}} \frac{k_{\mathrm{B}} T \sqrt{\lambda} K_{0}}{A_{1} \sqrt{L_{0}}}\left[1+\mu\left(K_{1}-\frac{1}{2} L_{1}\right)-\mu^{2}\left(\frac{1}{2} L_{2}-\frac{3}{8} L_{1}^{2}+\frac{1}{2} L_{1} K_{1}\right)\right]+O\left(\mu^{3}\right), \\
D\left(m_{2}\right) & \simeq \frac{1}{m_{1}} \frac{k_{\mathrm{B}} T \sqrt{\lambda} \widetilde{K}_{0}}{A_{1} \sqrt{\widetilde{L}_{0}}}\left[1+\mu\left(\widetilde{K}_{1}-\frac{1}{2} \widetilde{L}_{1}\right)-\mu^{2}\left(\frac{1}{2} \widetilde{L}_{2}-\frac{3}{8} \widetilde{L}_{1}^{2}+\frac{1}{2} \widetilde{L}_{1} \widetilde{K}_{1}\right)\right]+O\left(\mu^{3}\right), \\
\lim _{\mu \rightarrow 0} \frac{D\left(m_{2}\right)}{D\left(m_{1}\right)} & =\frac{\widetilde{K}_{0}}{K_{0}} \sqrt{\frac{L_{0}}{\widetilde{L}_{0}}}=\sqrt{\frac{A_{1} C_{1}-B_{1}^{2}}{A_{1} \widetilde{C}_{3}-\widetilde{B}_{2}}\left(\frac{\widetilde{B}_{2}}{B_{1}-A_{1}^{2}}\right)^{3 / 2} .}
\end{aligned}
$$

\section{Results and discussion}

In the previous section we have shown that for small mass ratio of light and heavy particles $\mu$ the self-diffusion coefficient can in general be presented in the form

$$
D\left(m_{i}\right)=\frac{k_{\mathrm{B}} T}{\sqrt{m_{1}}} \cdot R_{0}^{(i)}\left(1+R_{1}^{(i)} \mu+R_{2}^{(i)} \mu^{2}+\cdots\right),
$$

where $R_{n}^{(i)}$ are some coefficients which are expressed via static correlation functions and do not depend on the masses.

We developed the hierarchy of approximations for $R_{n}^{(i)}$ that is expected to tend to an exact result from above and below when the order of approximations increases. This prediction is based on the qualitative behaviour of the results obtained by Tankeshwar and Sharma [10] and on the work by Hachkevych and Mryglod [20] where the same approach is used to describe the generalized hydrodynamics of a simple fluid in a wide range of wave vectors and frequencies. Also we plan to test it in molecular dynamics.

As to the advantages of our representation, first of all, we remind that mass-dependence is extracted from expressions for velocity correlation functions analytically. We do not guess the kind of dependence from MD simulation results and do not use any interpolation techniques. No other approximations except for the Taylor expansion are used within the memory function formalism.

Representation (38) provides a useful possibility to analyze the self-diffusion coefficients for a wide range of mass ratios. Once the coefficients $R_{n}^{(i)}$ for a particular interaction potential $U(r)$ were calculated (for instance, by means of MD simulations), they can be used to determine the self-diffusion coefficients for a set of $\mu$ changing from 0 to 1 . Of course, for $\mu$ close to 1 we cannot use the Taylor expansion and intermediate expressions for $\Gamma_{n}$ from equations (22)-(27) should be used. But for smaller $\mu$ we can estimate how many terms in equation (38) should be taken into account to obtain the desired precision.

Moreover, since the coefficients $R_{n}^{(i)}$ do not contain masses in their definition, no finite-size effect caused by different time scales for heavy and light particles has to be accounted for.

The obtained expression has a correct Brownian limit for the second and higher-order approximations

$$
\lim _{\mu \rightarrow 0} \frac{D\left(m_{2}\right)}{D\left(m_{1}\right)}=\frac{R_{0}^{(2)}}{R_{0}^{(1)}} .
$$

Note that the coefficients $R_{0}^{(1)}$ and $R_{0}^{(2)}$ contain only a subset of the whole variety of coefficients $A_{1}, \ldots, \widetilde{C}_{3}$. Namely, for the second approximations these are $A_{1}, B_{1}$ and $\widetilde{B}_{2}$ (see equation (34)) and for the third approximations this set is supplemented with $C_{1}$ and $\widetilde{C}_{3}$ (equation (37)). In other words, in the Brownian limit only the coefficients in terms with the highest power of $m_{1}^{-1}$ (i. e. the lowest power of $m_{2}^{-1}$ ) in the expression for frequency sum rules (16)-(21) are significant.

An additional way of improving our approximations is to vary the parameter $\lambda$ in (9). The value of $\lambda$ in a particular approximation which provides the less deviation from MD data (e. g. 
calculated using Green-Kubo relation or via the mean square displacement) should be picked out. It can also be chosen as the initial estimate in other approximations.

\section{Conclusion}

In the present paper we have studied the mass dependence of the self-diffusion coefficient of light and heavy particles of binary fluid mixture. In particular, we have investigated the case of Brownian limit, i. e. when the mass of light particles is fixed and the mass of heavy particles tends to infinity. We have used general Green-Kubo relation for the self-diffusion coefficient, and then the memory function formalism to obtain recurrent relations for the velocity autocorrelation functions. These functions were expressed via static correlation functions with the mass dependence being separated.

We obtained a general relation for the self-diffusion coefficients which show polynomial dependence on the mass ratio of light and heavy particles $\mu$. In the limit of $\mu \rightarrow 0$ the self-diffusion coefficients of light and heavy particles proved to be independent of the mass of heavy particles. This is an important result since neither equation (1) nor equation (2) provide good limit for this case. We developed the hierarchy of approximations for the self-diffusion coefficients that tends to the exact result from above and below when the order of approximations increases.

There are several possible ways of extending the results presented here. First of all the results are planned to be tested in molecular dynamics. Also we are interested in studying other transport coefficients such as shear viscosity, thermal conductivity or mutual diffusion. In particular, shear viscosity can be calculated directly from the Stokes-Einstein relation.

\section{References}

1. Nuevo M.J., Morales J.J., Heyes D.M., Phys. Rev. E, 1995, 51, 2026-2032.

2. Nuevo M.J., Morales J.J., Heyes D.M., Phys. Rev. E, 1998, 58, 5845-5854.

3. Snook I.K., O'Malley B., McPhie M.G., Daivis P.J., J. Mol. Liq., 2003, 103-104, 405-421.

4. McPhie M.G., Daivis P.J., Snook I.K., Phys. Rev. E, 2006, 74, 031201.

5. Ould-Kaddour F., Levesque D., Phys. Rev. E, 2000, 63, 011205.

6. Ould-Kaddour F., Levesque D., J. Chem. Phys., 2007, 127, 154514.

7. Bearman R.J., Jolly D.L., Mol. Phys., 1981, 44, 665.

8. Tankeshwar K., Phys. Chem. Liq., 1991, 24, 21-29.

9. Tankeshwar K., J. Phys.: Condens. Matter, 1995, 7, 9715-9722.

10. Sharma R., Tankeshwar K., Sharma K.C., Phys. Rev. E, 1999, 59, 460-468.

11. Bryk T., Mryglod I., J. Phys.: Condens. Matter, 2005, 17, 413-427.

12. Fenz W., Mryglod I.M., Prytula O., Folk R., Phys. Rev. E, 2009, 80, 021202.

13. Rull L.F., de Miguel E., Morales J.J., Nuevo M.J., Phys. Rev. A, 1989, 40, 5856-5859.

14. Toxvaerd S., J. Chem. Phys., 1984, 81, 5131-5136.

15. Bogolyubov M. M. Problems of Dynamical Theory in Statistical Physics. Gostekhizdat, MoskowLeningrad, 1946 (in russian).

16. Boon J.P., Yip S. Molecular Hydrodynamics. McGraw Hill, New York, 1979.

17. Fushiki M., Phys. Rev. E, 2003, 68, 021203.

18. Mori H., Prog. Theor. Phys., 1965, 33, 423-455.

19. Lee L.L., Physica A, 1980, 100, 205-211.

20. Mryglod I.M., Hachkevych A.M., Ukrainian Journal of Physics, 1999, 44, 901-907. 


\title{
Залежність коефіцієнтів самодифузії від маси у бінарних плинах із сильною масовою асиметрією
}

\author{
І.М. Бінас, І.М. Мриглод \\ Інститут фізики конденсованих систем НАН України, вул. Свєнціцького, 1, 79011 Львів, Україна \\ Отримано 24 червня 2009 р., в остаточному вигляді - 9 вересня 2009 р.
}

\begin{abstract}
Досліджуються коефіцієнти самодифузії бінарних плинів із компонентами, що відрізняються лише масами, зокрема вивчається випадок, коли співвідношення мас легких і важких частинок $\mu$ прямує до нуля. Ці коефіцієнти розраховані за допомогою формалізму функцій пам'яті з використанням систематичної послідовності апроксимацій для часів релаксації автокореляційної функції швидкостей. Отримано загальний вираз для коефіцієнтів самодифузії, що демонструють поліноміальну залежність від співвідношення мас $\mu$. Отриманий вираз має коректну броунівську границю. Розроблено ієрархію апроксимацій для коефіцієнтів самодифузії, що при збільшенні порядку апроксимацій прямують до точного розв'язку зверху і знизу відповідно.
\end{abstract}

Ключові слова: самодифузія, масова асиметрія, бінарні флюїди, броунівська границя

PACS: $66.10 .-x$ 